\title{
Influence of Flame Front on the Flow Field
}

\author{
By H. S. TSIEN, ${ }^{1}$ PASADENA, CALIF.
}

Flame front is a region in the flow field where rapid change in the chemical composition of the fluid occurs with consequent release of chemical energy in the form of heat. In the majority of cases the phenomenon is a very complicated one involving the heat transfer by conduction and radiation, the changes in concentration of the different components by diffusion and chemical reaction. Ow ing to this and the difficult problem of chemical kinetics, only recently the complete theory of flame front has been formulated, particularly by the group under J. O. Hirschfelder. ${ }^{2}$ Fortunately, as a result of the rapid rate of chemical reaction, the thickness of the flame front under ordinary conditions is generally very small, being less than $1 \mathbf{~ m m}$. Therefore, if one is interested in the influence of flame front on the flow field but not on the detailed structure of the flame, the flame can be assumed as infinitesimally thin, and only the final changes of the state of fluid due to combustion need be considered. This procedure is entirely analogous to that of treating the shock wave as having zero thickness in studying dynamics of compressible fluids. This simplification will be adopted for the present inve stigation.

$\mathrm{N}$ EGLECTING the change in specific heats of the gas by combustion and assuming perfect gas, very simple relations for quantities before the combustion and after the combustion can be obtained. This will be determined first. With these relations, the production of vorticity due to nonuniform condition before the flame front will be studied. After these preliminary results, the problem of flame width in a twodimensional combustion chamber of constant width with a flame holder at the center will be solved approximately. This problem was first solved by A. C. Scurlock. ${ }^{3}$ The present calculation is, however, very much simpler and is extended to take into account the compressibility of the gas. The effect of compressibility gives an anomalous spreading of the flame in the channel, and its significance in the efficiency of combustion and combustion-chamber design will be discussed.

\section{Flame Front}

Consider the flame front to be stationary, and the unburned gas flows into it with a normal velocity $S$ and leaves it with a

${ }^{1}$ Robert H. Goddard Professor of Jet Propulsion, Daniel and Florence Guggenheim Jet Propulsion Center, California Institute of Technology. Mem. ASME

${ }_{2}$ "Theory of Propagation of Flames. Part I. General Equations," by J. O. Hirschfelder and C. E. Curtiss, Third Symposium on Combustion, Flames and Explosion Phenomena, Williams and Wilkins, Baltimore, Md., 1949, pp. 121-127.

"Theory of Flame Propagation," Journal of Chemical Physics, vol. 17,1949, p. 1076.

3 "Flame Stabilization and Propagation in High-Velocity Gas Streams," by A. C. Scurlock, Meteor Report no. 19, Massachusetts Institute of Technology, 1948

Presented at the 1950 Heat Transfer and Fluid Mechanies Institute, Los Angeles, Calif., June 28-30, 1950.

Discussion of this paper should be addressed to the Secretary. ASME, 29 West 39 th Street. New York, N. Y., and will be accepted until July 10,1951 , for publication at a later date. Difcussion received after the closing date will be returned.

Note: Statements and opinions advaneed in papers are to be understood as individual expressions of their authors and not those of the Society. Manuscript received by the Applied Mechanics Division, October 23, 1950. velocity $w_{2} ; S$ is then the normal flame velocity. Let $p, \rho$, and $\gamma$ be the pressure, density, and the ratio of specific heats, respectively. The subscript 1 will denote quantities before the combustion and the subscript 2 after the combustion. Then the equation of continuity is

$$
\rho_{1} S=\rho_{2} w_{2}
$$

The momentum equation is

$$
\rho_{1} S^{2}+p_{1}=\rho_{2} w_{2}^{2}+p_{2}
$$

If $\lambda$ is the ratio of the stagnation temperature after combustion to the stagnation temperature before the combustion, the energy equation is

$$
\lambda\left[\frac{1}{2} S^{2}+\frac{\gamma}{\gamma-1} \frac{p_{1}}{\rho_{1}}\right]=\frac{1}{2} w_{2}^{2}+\frac{\gamma}{\gamma-1} \frac{p_{2}}{\rho_{2}} \ldots \ldots
$$

Consider the quantities $\rho_{1}, S$, and $\lambda$ as given by the detailed theory of flame or by experiment. Then Equations [1], [2], and [3] are three equations for the three unknowns $w_{2}, p_{2}$, and $\rho_{2}$. The solution corresponding to normal burning can be written as follows

$$
\begin{aligned}
& \frac{w_{2}}{S}=\frac{\rho_{1}}{\rho_{2}}=\lambda+\frac{\gamma+1}{2} \lambda(\lambda-1) M_{1}{ }^{2} \\
& +\frac{\gamma+1}{2} \lambda(\lambda-1)\{1+(\gamma+1)(\lambda-1)\} M_{1}^{4}+\ldots \ldots \ldots[4 \\
& \frac{p_{2}}{p_{1}}=1-\gamma(\lambda-1) M_{1}^{2}-\frac{\gamma+1}{2} \gamma \lambda(\lambda-1) M_{1}{ }^{4} \\
& -\frac{\gamma+1}{2} \gamma \lambda(\lambda-1)\{1+(\gamma+1)(\lambda-1)\} M_{1}{ }^{6}+\ldots \ldots[5
\end{aligned}
$$

The temperature ratio $T_{2} / T_{1}$ is

$\frac{T_{2}}{T_{1}}=\lambda-\frac{\gamma-1}{2} \lambda(\lambda-1) \mathbf{M}_{1}{ }^{2}-\frac{\gamma^{2}-1}{2} \lambda^{2}(\lambda-1) \mathbf{M}_{1}{ }^{4}+\ldots$

In these equations, the quantity $M_{1}$ is the ratio of the flame velocity $S$ to the sound velocity $a_{1}$ in gas before the combustion, or the Mach number of the flame. Since, under ordinary conditions, $S$ is of the order of $1 \mathrm{fps}$ while $a_{1}$ is of the order of $1000 \mathrm{fps}, M_{1}$ is very small and generally only the first terms in Equations [4], [5], and [6] are necessary.

\section{Production of Vorticity by Flame}

It is known that in a nonviscous and non-heat-conducting fluid, if the pressure is only a function of density, then the vorticity of any fluid element is a constant. These conditions of flow are satisfied approximately by real fluid without heat addition or combustion. Most fluid motions of practical in terest originate from a uniform state, where vorticity is zero or the motion is irrotational. Then the motion will remain irrotational. This irrotationality of flow simplifies greatly the analysis of the field. Hence it is of interest to investigate the extent to which this condition is destroyed by flame front. In other words, the production of vorticity by flame should be calculated.

For simplicity, consider the two-dimensional flow. Let the gas be of uniform composition and having a constant sum of en- 
thalpy and the kinetic energy, or isoenergetic, before combustion. In view of the generally irrotational flow without combustion, the flow before the flame front will be assumed to be irrotational and thus isentropic. The problem specifically is then to calculate the vorticity $\omega$ after the flame. Let $\sigma$ be the specific entropy and $\psi$ the stream function. Then it is known ${ }^{4}$ that for steady flows

$$
\omega=\rho_{2} T_{2} \frac{d \sigma_{2}}{d \psi_{2}}
$$

where again the subscript 2 denotes quantities after the flame front. For perfect gas, Equation [7] can be written as

$\omega=\frac{1}{\gamma-1} p_{2} \frac{d}{d \psi_{z}}\left\{\log \left(\frac{p_{2}}{p_{1}}\right)-\gamma \log \left(\frac{\rho_{2}}{\rho_{1}}\right)+\log \frac{p_{1}}{\rho_{1}{ }^{2}}\right\} \ldots$

Before the flame, $\sigma_{1}$ is constant, so $p_{1} / \rho_{1}^{\gamma}$ is constant. Since $d \psi_{2}=d \psi_{1}$, Equation [8] simplifies to

$$
\omega=\frac{1}{\gamma-1} p_{1}\left(\frac{p_{2}}{p_{1}}\right) \frac{d}{d \psi_{1}}\left\{\log \left(\frac{p_{2}}{p_{1}}\right)+\gamma \log \left(\frac{\rho_{1}}{\rho_{2}}\right)\right\}
$$

The pressure ratio $p_{2} / p_{1}$ and the density ratio $\rho_{2} / \rho_{1}$ are given by Equations [4] and [5]. Therefore the production of vorticity is controlled by the variation of the flame Mach number $\mathbf{M}_{\mathbf{1}}$ or $S / a_{1}$ and the parameter $\lambda$ along the flame front.

Perhaps due to very intense transport phenomena generated by the large temperature rise in the flame, the normal flame velocity $S$ is observad to be only weakly dependent upon the local conditions before combustion. According to H. Sachsse, ${ }^{5}$ the normal flame velocity of methane-oxygen mixture is increased to 3 times the value at room temperature by preheating the mixture to $1000 \mathrm{C}$. Later experiments by Sachsse and E. Bartholomé $e^{6}$ indicated an increase of approximately 30 per cent in flame velocity by preheating various gas mixtures from 20 to $100 \mathrm{C}$. From this evidence, it seems that the normal flame velocity increases roughly as the absolute temperature of the "unburned" gas mixture. The experiments on the influence of pressure on the flame velocity do not seem to give conclusive results, but in any event, the influence is not large. Therefore, for the computation of the production of vorticity by flame front, two separate cases can be considered. For the first case, the flame speed $S$ is taken to be a constant. For the second case, the flame speed $S$ is to be proportional to the absolute tomperature $T_{1}$.

Write

$$
\left.\begin{array}{l}
\frac{p_{2}}{p_{1}}=F \\
\frac{\rho_{1}}{\rho_{2}}=G
\end{array}\right\}
$$

Then Equation $[9]$ can be written as

$$
\begin{aligned}
\omega=\frac{1}{\gamma-1} F & {\left[\left\{\frac{1}{F} \frac{\partial F}{\partial \mathbf{M}_{1}{ }^{2}}+\frac{\gamma}{G} \frac{\partial G}{\partial \mathbf{M}_{1}{ }^{2}}\right\}\left(p_{1} \frac{d \mathbf{M}_{1}^{2}}{d p_{1}}\right)\right.} \\
& \left.+\left\{\frac{1}{F} \frac{\partial F}{\partial \lambda}+\frac{\gamma}{G} \frac{\partial G}{\partial \lambda}\right\}\left(p_{1} \frac{d \lambda}{d p_{1}}\right)\right] \frac{d p_{1}}{d \psi_{1}} \ldots . .
\end{aligned}
$$

\footnotetext{
4 "On Rotational Gas Flows," by A. Vazsonyi, Quarterly of Applied Mathematics, vol. 3, 1945, pp. 29-37.

5 "Über die Temperaturabhängigkeit der Flammengeschwindigkeit und das Temperaturgefälle in der Flammenfront," by H. Sachsse, Zeitschrift für physikalische Chemie (A), vol. 180, 1937, pp. 305313 .

6 "Beiträge zur Frage der Flammengeschwindigkeit," by $\mathrm{H}$. Sachsse and E. Bartholomé, Zeitschrift für Elektrochemie und angewandte physikalische Chemie, vol. 53, 1949, pp. 183-190.
}

If $\Delta H$ is the heat addition per unit mass of the gas due to chemical reaction, then from the dafinition of $\lambda$

$$
\lambda=\frac{\Delta H}{\frac{1}{2} S^{2}+\frac{\gamma}{\gamma-1} \frac{p_{1}}{\rho_{1}}}+1
$$

Therefore $\lambda$ is not a constant, in spite of the fact that the heat released $\Delta H$ can be considered as a constant with good approximation.

For the first case, $S$ is a constant, and

$$
M_{1}^{2}=\frac{S^{2}}{a_{0}^{2}}\left(\frac{a_{0}}{a_{1}}\right)^{2}=\frac{S^{2}}{a_{0}^{2}}\left(\frac{p_{0}}{p_{1}}\right)^{\frac{\gamma-1}{\gamma}}
$$

where the subscript 0 refers to the stagnation condition before the flame. Then

$$
p_{1} \frac{d \mathbf{M}_{\mathbf{l}}^{2}}{d p_{1}}=-\frac{\gamma-1}{\gamma} \frac{S^{2}}{a_{1}^{2}}=-\frac{\gamma-1}{\gamma} \mathbf{M}_{1}^{2} \ldots \ldots
$$

Similarly

$$
p_{\mathrm{l}} \frac{d \lambda}{d p_{1}}=-\frac{\gamma-1}{\gamma}(\lambda-1) \frac{1}{1+\frac{\gamma-1}{2} M_{1}^{2}} \ldots
$$

For the second case, $S$ is proportional to the temperature $T_{1}$ or to $a_{1}{ }^{2}$. Then

$$
M_{1}^{2}=\frac{S_{0}^{2}}{a_{0}^{2}}\left(\frac{a_{1}}{a_{0}}\right)^{2}=\frac{S_{0}^{2}}{a_{0}^{2}}\left(\frac{p_{1}}{p_{0}}\right)^{\frac{\gamma-1}{\gamma}}
$$

Therefore

$$
p_{1} \frac{d \mathbf{M}_{1}^{2}}{d p_{1}}=\frac{\gamma-1}{\gamma} \frac{S_{0}^{2}}{a_{0}^{2}}\left(\frac{a_{1}}{a_{0}}\right)^{2}=\frac{\gamma-1}{\gamma} \mathbf{M}_{1}^{2} \ldots \ldots
$$

The corresponding derivative of $\lambda$ is

$$
p_{1} \frac{d \lambda}{d p_{1}}=-\frac{\gamma-1}{\gamma}(\lambda-1) \frac{1+(\gamma-1) \mathbf{M}_{1}^{2}}{1+\frac{\gamma-1}{2} M_{1}{ }^{2}} \cdots
$$

The derivative of pressure $p_{1}$ with respect to $\psi_{1}$ can be expressed in a more convenient form: If $q_{1}$ is the magnitude of velocity immediately ahead of the shock, $n$ the normal distance from streamline to streamline, then

$$
d \psi_{1}=\rho_{1} q_{1} d n
$$

Furthermore, the balance of centripetal forces by pressure requires

$$
\frac{d p_{1}}{d n}=-\frac{\rho_{1} q_{1}^{2}}{R_{1}}
$$

where $R_{1}$ is the radius of curvature of the streamline immediately ahead of the shock, positive when the streamline is concave with respect to positive direction of $q_{1}$. From these two relations

$$
\frac{d p_{1}}{d \psi_{1}}=-\frac{q_{1}}{R_{1}} \ldots \ldots \ldots \ldots \ldots \ldots
$$

By substituting Equations [13], [14], and [17] into Equation [11], and by using Equations [4] and [5], the vorticity $\omega$ generated by the flame front for the case of constant flame speed $S$ can be determined. By substituting Equations [15], [16], and [17] into Equation [11], the vorticity $\omega$ for the case of variable flame speed can be computed.

However, it is important to note that the value of the flame 
speed is generally so small as to make $M_{1}$ negligible compared with unity. Then $p_{1}\left(d \mathbf{M}_{1}{ }^{2} / d p_{1}\right)$ is negligibly small in comparison with $p_{1}\left(d \lambda / d p_{1}\right)$ and the latter is approximately the same for both eases, i.e.

$$
p_{1} \frac{d \lambda}{d p_{1}} \cong-\frac{\gamma-1}{\gamma}(\lambda-1) \quad \text { for } \quad M_{1}^{2} \ll 1 \ldots
$$

By making the same approximation for the functions of $F$ and $G$ and their derivatives, the vorticity generated $\omega$ is simply

$$
\omega \cong \frac{q_{1}}{R_{1}}\left(\frac{\lambda-1}{\lambda}\right) \text { for } \quad M_{1}^{2} \ll 1 \ldots \ldots \ldots
$$

It is seen from Equation [19] that when $R_{1} \rightarrow \infty, \omega \rightarrow 0$ as expected. Furthermore, when no combustion occurs, no heat is added, and $\lambda=1$, then $\omega=0$. But when there is combustion, the combustion will generate appreciable vorticity of the order of $q_{1} / R_{1}$.

\section{Flame Width in a Uniform Channel}

The problem of spreading of the flame in a homogeneous premixed combustible from an idealized point flame holder located at the axis of a two-dimensional uniform channel, Fig. 1, was

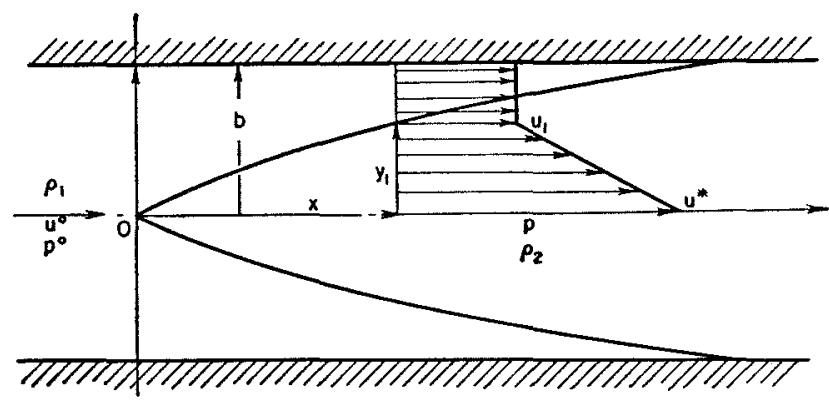

Fig. 1 Flame Propagation in a Two-Dimensional Combustion Chamber of Constant Width From the Point Flame Holden 0

first solved by A. C. Scurlock. ${ }^{3}$ For interpreting the experimental data, he needs the relation between the flame width $y_{1}$ and the fraction of gas burned. He assumes for simplicity of calculation, that the fluid is a perfect incompressible fluid. The assumption of incompressible flow is justified on the ground that the velocity of flow is small compared with the speed of sound. This means then the flame Mach number $M_{1}$ is negligibly small. From Equations [4], [5], and [6], it is seen that

$$
\frac{\rho_{1}}{\rho_{2}} \approx \lambda, \quad \frac{p_{2}}{p_{1}} \cong 1
$$

Therefore under the assumption of incompressible flow, the effect of combustion is to change the density by a factor of $\lambda$ (the ratio of stagnation temperatures) while the pressure remains constant. Scurlock then observed that since he is interested in cases where the flow velocities are very much larger than the normal flame velocity $S$, the flame fronts will be inclined at small angles from the channel axis. The result is that all streamlines are nearly parallel to the channel axis. Then as an approximation, the magnitude of velocity at any point is taken to be the magnitude of $x$-component $u$ (parallel to the channel axis) of velocity, and neglect the effects of the curvature of the streamlines. If curvature of the streamlines is neglected, the pressure variation in the $y$-direction (normal to the channel axis) due to centrifugal forces must be also neglected. Equation [20] further shows that there is no pressure change by crossing the flame front; then it is evident that the pressure $p$ in any cross section of the channel must be constant, whether in the unburned gas or the burned gas. This means the pressure is continuous in the whole field. Therefore the velocity $u$ must also be continuous by crossing the flame front.

The entire problem is then reduced to a quasi-one-dimensional calculation: The fluid density is constant in respective regions of unburned and burned regions. The ratio of densities is $\lambda$. The unburned gas flows with constant uniform velocity $u^{0}$ and density $\rho_{1}$ until it reaches the section containing the flame holder 0, Fig. 1. The gas immediately after the flame holder has still the same velocity $u^{0}$ but a density $\rho_{2}=\rho_{1} / \lambda$. At a section $x$ downstream of the flame holder, the velucity at the channel axis is increased to $u^{*}$ and the velocity in the unburned gas, uniform in the unburned region of the section, is increased from $u^{0}$ to $u_{1}$. The pressure $p$ at $x$ is, however, smaller than the pressure $p^{0}$ of the approaching unburned gas. By using Bernoulli's theorem

$$
\left.\begin{array}{l}
\frac{1}{2} \rho_{1}\left(u_{1}^{2}-u^{0^{2}}\right)=p^{0}-p \\
\frac{1}{2} \rho_{2}\left(u^{*^{2}}-u^{0^{2}}\right)=p^{0}-p
\end{array}\right\} \ldots \ldots \ldots
$$

Therefore by eliminating $p$

$$
\frac{u^{*}}{u^{0}}=\sqrt{1+\lambda\left(\frac{u_{1}^{2}}{u^{0^{2}}}-1\right)}
$$

This equation shows that $u^{*}$ is always larger than $u_{1}$.

At the section $x$, the velocity $u$ in the burned region decreases from the value $u^{*}$ at the axis to $u_{1}$ at the flame front $y=y_{1}$. Scurlock, ${ }^{4}$ using a laborious numerical method, has computed the velocity profile for various values of $\lambda$. Fig. 2 is taken from his paper. The accuracy of the result is, of course, predicated by the assumptions. That it cannot be exact is seen by using the result of the previous section. Along the axis, the curvature of streamline is zero. From Equation [19], the vorticity $\left(\partial_{v} / \partial x-\right.$ $\partial u / \partial y$ ) along the axis is then always zero. Furthermore the $y$ component of velocity $v$ is by symmetry zero along the axis. Therefore $\partial v / \partial x$ is zero along the axis. The $\partial u / \partial y$ must be also zero along the axis. This is not so in Scurlock's result. This discrepancy must be, nevertheless, localized. In gross features then, Scurlock's results are accurate for the purpose of flamewidth determinations.

On the other hand, if gross features are the only results that can be expected from the simplified quasi-one-dimensional calculation, the calculation could be made very much simpler: Take the velocity profile from $y=0$ to $y=y_{1}$ to be linear. Then with Equation [22]

$$
\begin{aligned}
\frac{u}{u^{0}}=\sqrt{1+\lambda\left(\frac{u_{1}^{2}}{u^{0^{2}}}-1\right)}-\left\{\sqrt{1+\lambda\left(\frac{u_{1}^{2}}{u^{0^{2}}}-1\right)}\right. & \\
& \left.-\frac{u_{1}}{u^{0}}\right\} \frac{y}{y_{1}} \ldots \ldots
\end{aligned}
$$

The condition that the same mass must flow through each section then specifies

$$
\rho_{2} \int_{0}^{y_{1}} u d y+\rho_{1}\left(b-y_{1}\right) u_{1}=\rho_{1} b u^{0} .
$$

where $b$ is the half width of the channel. By substituting Equation $[23]$ into [24] and by observing $\rho_{1} / \rho_{2}=\dot{\lambda}$

$$
\frac{1}{2 \lambda}\left(\frac{y_{1}}{b}\right)\left[\sqrt{\left(1+\lambda \frac{u_{1}^{2}}{u^{0^{2}}}-1\right)}+\frac{u_{1}}{u^{0}}\right]+\frac{u_{1}}{u^{0}}\left(1-\frac{y_{1}}{b}\right)=1
$$

By solving for $\left(y_{1} / b\right)$, denoted by $\eta$, one has the simple relation 


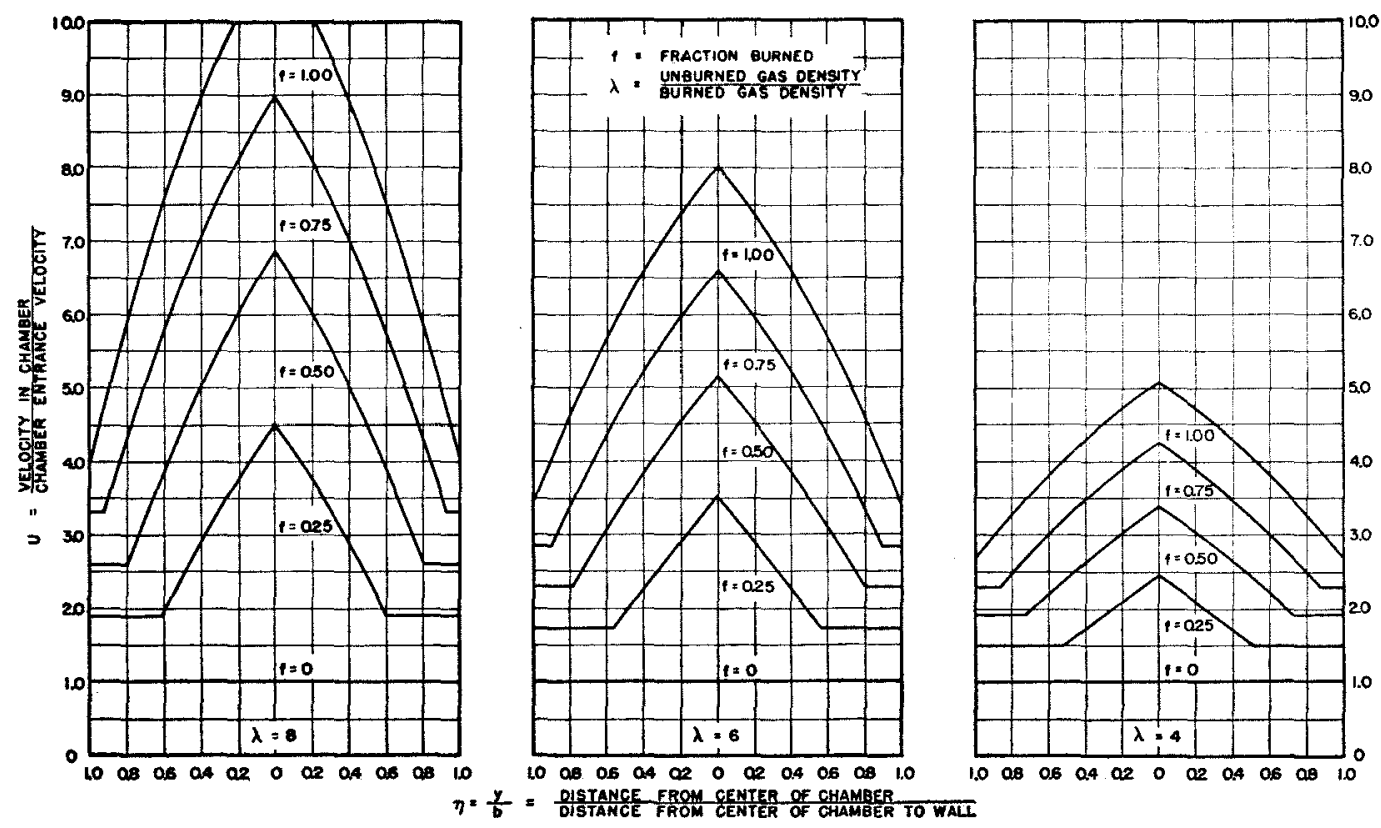

Frg. 2 Velocity Profile in Combustion Chamber With Different Flame Width and at Various Density Ratios (Computed by A. C. Scurlock, reference 3.)

where

$$
\eta=\frac{y_{1}}{b}=\frac{U-1}{\left(1-\frac{1}{2 \lambda}\right) U-\frac{1}{2 \lambda} \sqrt{1+\lambda\left(U^{2}-1\right)}}
$$

$$
U=u_{1} / u^{0}
$$

The fraction of the gas burned $f$ is

$$
f=1-\frac{\rho_{1}\left(b-y_{1}\right) u_{1}}{\rho_{1} b u^{0}}=1-U(1-\eta)
$$

By combining Equation [25] with [26]

$$
f=\frac{(U-1)\left[\sqrt{1+\lambda\left(U^{2}-1\right)}+U\right]}{(2 \lambda-1) U-\sqrt{1+\lambda\left(U^{2}-1\right)}} .
$$

Equations [25] and [27] can be considered as the parametric representation of the relation between the nondimensional flame width $\eta$ and the fraction burned $f$. Computations ${ }^{7}$ using these equations have been carried out for $\lambda=4,6$, and 8 . The results are compared with Scurlock's results in Fig. 3 . The agreement is satisfactory. It seems then there is no need for the complicated numerical procedure of Scurlock. ${ }^{8}$

\section{Effect of Compressibility on Flame Width}

Since the cases of interest are cases where the normal flame speed is small compared with the gas velocity, it is reasonable to speak of compressible flow of gas in general while still considering the flame Mach number $M_{1}$ in Equations [4], [5], and [6] to be negligibly small. Then the conclusions drawn in the previous section about changes of density and pressure in crossing the flame front still hold. In particular, the temperature of the gas is increased by a factor $\lambda$ in crossing the flame holder. The $\lambda$ at different points of the flame front is not the same as shown by Equation [12]. However, $\lambda$ will be taken to be a constant

The author is indebted to Mr. D. Shonerd for carrying out the numerical computations in this paper.

${ }^{8}$ In the Appendix, a complete mathematical formulation of Scurlock's problem is given.

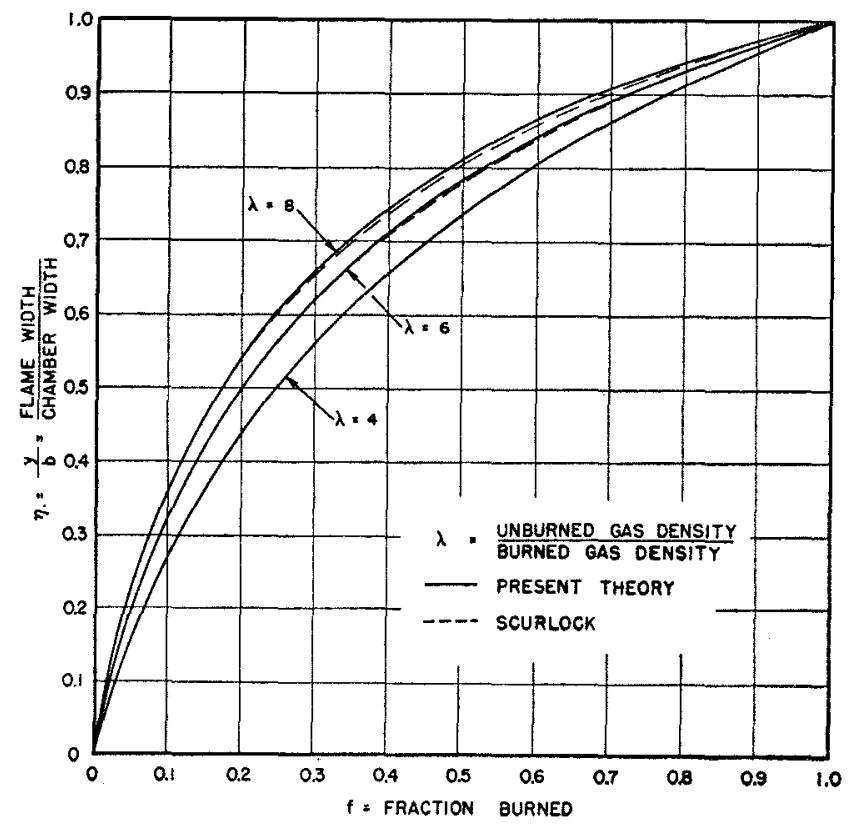

Fig. 3 Flame Width as Function of Fraction Burned at Various Density Ratios $\lambda$, for Incompressible Flutid

as an approximation. Only here Bernoulli's equation must be modified for the compressibility effects. Therefore, in place of Equation [21]

$$
\left.\begin{array}{l}
\frac{1}{2} u^{*^{2}}+c_{p} T^{*}=\frac{1}{2} u^{0^{2}}+c_{p} \lambda T^{0} \\
\frac{1}{2} u_{\mathrm{I}}{ }^{2}+c_{p} T_{\mathbf{1}}=\frac{1}{2} u^{0^{2}}+c_{p} T^{0}
\end{array}\right\}
$$

where $T^{\mathbf{0}}, T_{1}$, and $T^{*}$ are the temperatures of the approaching unburned gas, the unburned gas at section $x$, and the burned gas at section $x$ and the channel axis; $c_{p}$ is the specific heat at con- 
stant pressure. Along each streamline, the entropy of gas is a constant in either the unburned region or the burned region. Therefore, in either region the corresponding isentropic relations hold. Equations [29] can then be modified to

$$
\begin{aligned}
& \frac{1}{2} u^{*^{2}}+\frac{\gamma}{\gamma-1} \frac{p^{0}}{\rho^{0}} \lambda\left(\frac{p}{p^{0}}\right)^{\frac{\gamma-1}{\gamma}}=\frac{1}{2} u^{0^{2}}+\frac{\gamma}{\gamma-1} \lambda \frac{p^{0}}{\rho^{0}} \\
& \frac{1}{2} u_{1}^{2}+\frac{\gamma}{\gamma-1} \frac{p^{0}}{\rho^{0}}\left(\frac{p}{p^{0}}\right)^{\frac{\gamma-1}{\gamma}}=\frac{1}{2} u^{0^{2}}+\frac{\gamma}{\gamma-1} \frac{p^{0}}{\rho^{0}}
\end{aligned}
$$

By eliminating the pressure ratio $p / p^{0}$, Equation [22] is again obtained. Thus the relation between the burned velocity $u^{*}$ at the axis and the unburned velocity $u_{1}$ is not modified by the compressibility.

If the linear velocity profile through the burned region is again assumed, Equation [23] remains true. However, now it is necessary to distinguish the density $\rho_{1}$ of the unburned gas at section $x$ from the density $\rho^{0}$ of the approaching unburned gas. This ratio is easily obtained as

$$
\frac{\rho_{1}}{\rho^{0}}=\left[1-\frac{\gamma-1}{2} M^{0^{2}}\left(U^{2}-1\right)\right]^{\frac{1}{\gamma-1}} .
$$

where $M^{0}$ is the Mach number of the approaching unburned gas, $u^{0} / a^{0}$, and $U$ is again $u_{1} / u^{0}$. By using the approximation that the density of fluid decreases by the constant factor $1 / \lambda$ after crossing the flame, the continuity condition is now

$$
\frac{\rho_{1}}{\lambda} \int_{0}^{y_{1}} u d y+\rho_{1}\left(b-y_{1}\right) u_{1}=\rho^{0} b u^{0} \ldots
$$

By using Equations [23] and [31], Equation [32] gives

$$
\eta=\frac{y_{1}}{b}=\frac{U-\left[1-\frac{\gamma-1}{2} M^{0^{2}}\left(U^{2}-1\right)\right]^{-\frac{1}{\gamma-1}}}{\left(1-\frac{1}{2 \lambda}\right) U-\frac{1}{2 \lambda} \sqrt{1+\lambda\left(U^{2}-1\right)}}
$$

The fraction burned $f$ is then

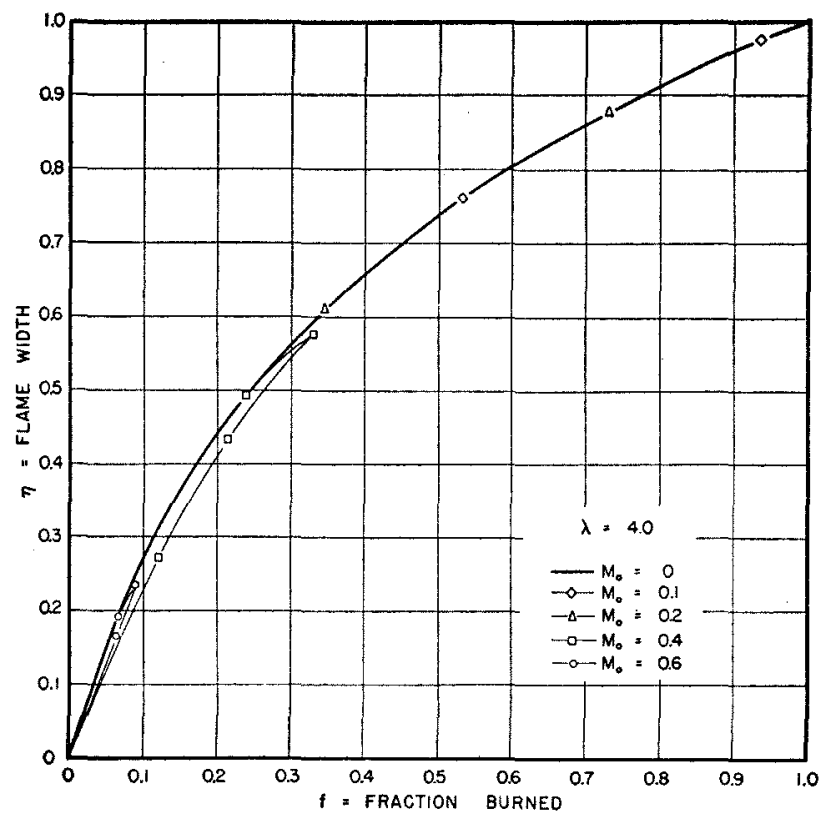

Fig. 4 Flame Width as Function of Fraction Burned at Density Ratro $\lambda=4$ for Various Initial Mach Numbers $M^{0}$

$$
f=1-\frac{\rho_{1}}{\rho_{0}} U(1-\eta)
$$

Equations [33] and [34] together with Equation [31] are the parametric representation of the relation between the nondimensional flame width $\eta$ and the fraction burned, $f$. The results of calculation are plotted in Figs. 4, 5, and 6. It is seen that the compressibility has little effect on the relation between the flame width $\eta$ and the fraction burned $f$. The curves at different approach Mach numbers $\mathbf{M}^{0}$ lie very close to the incompressibleflow curve calculated by using Equations [25] and [28]. Therefore the procedure adopted by Scurlock in using the incompressible curve for all his computations is indeed justified. Figs. 4,5 , and 6 , however, show another very important feature of the

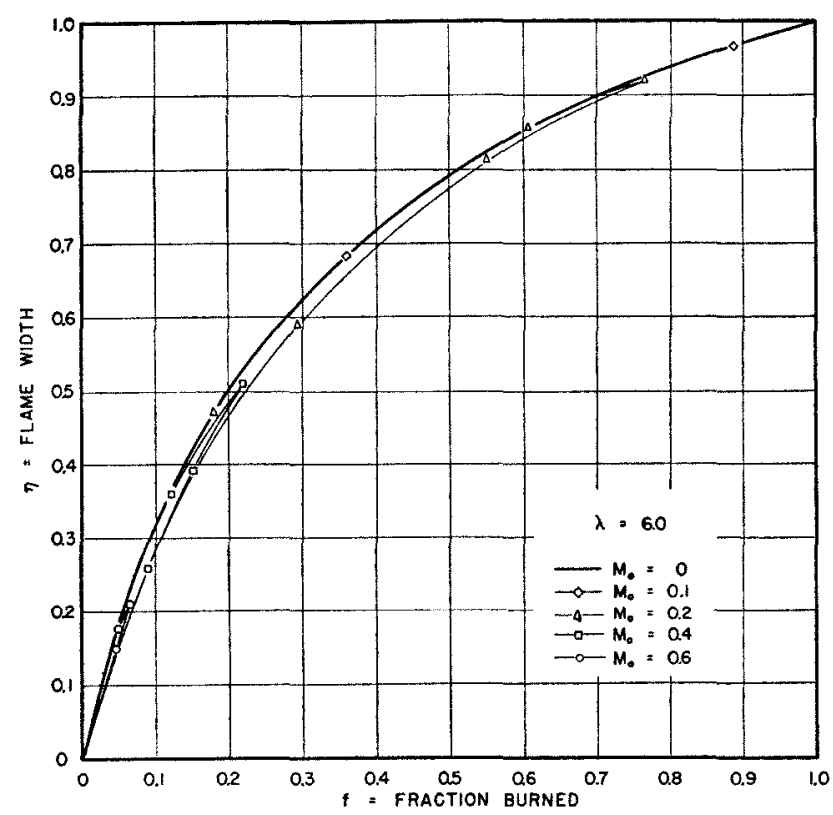

Fig. 5 Flame Width as Function of Fraction Burned at Density Ratio $\lambda=6$ for Vartovg Inttial Mach Numbers $M^{0}$

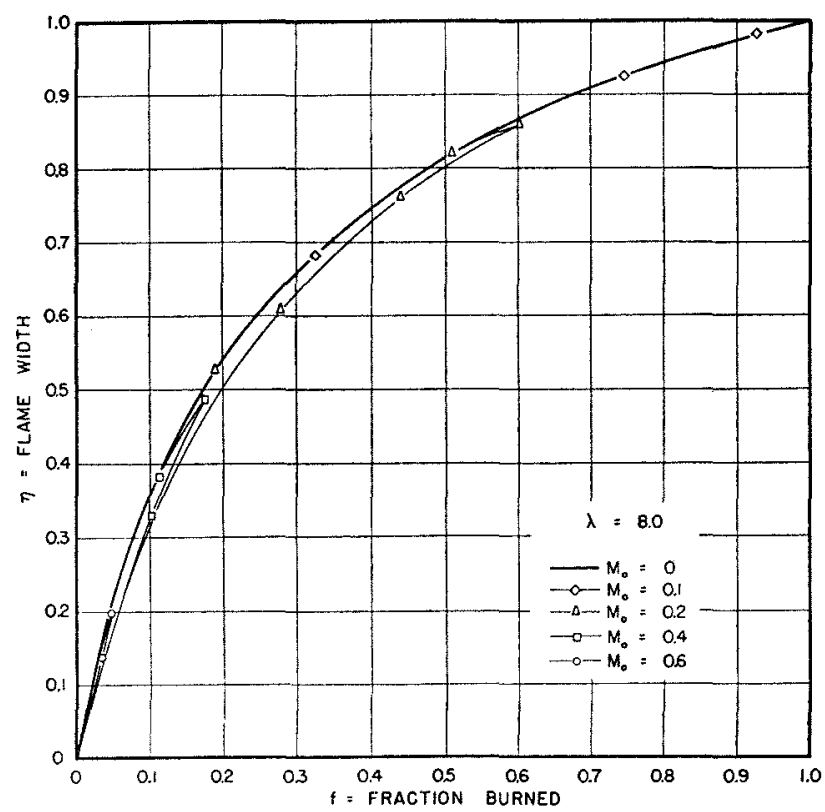

Ftg. 6 Flame Width as Function of Fraction Burned at Density Ratio $\lambda=8$ for Variovs Initial Mach Numbers $\mathbf{M}^{0}$ 
problem: Both the flame width $\eta$ and the fraction burned $f$, have maximum values at higher $M^{0}$. For $M^{0}=0.4$, the maximum flame width is only $1 / 2$ of the channel width and the maximum amount of gas burned is only $1 / 3$ of the input. At higher values of $M^{0}$, these fractions are even smaller. This definitely shows that for a combustion chamber of constant width, as assumed in the present analysis, it will be difficult if not impossible to have complete combustion at high flow velocities, even with a good flame holder to initiate the flame.

What is the physical situation which causes this anomaly? By crossing the flame front the flow velocity is maintained, but the temperature of the gas is increased by $\lambda$ times. Therefore the Mach number of the gas is reduced by combustion. In other words, the Mach number of the unburned gas is always higher than the Mach number of the burned gas. With equal reduction in pressure, the stream tube will contract less if the Mach number is higher. In fact, for supersonic flow, the stream tube will expand instead of contract when the pressure is reduced. Therefore the effect of compressibility of the gas is to make the width of the unburned gas relatively larger than the width for incompressible fluid. The effect is more prominent when the initial Mach number is higher. Therefore there will be one initial Mach number $\mathbf{M}_{c}{ }^{0}$, called the critical Mach number, for a given value of $\lambda$, at which

$$
\frac{d \eta}{d U}=0 \quad \text { at } \quad \eta=1, \quad U=U_{c}
$$

If the initial Mach number $\mathbf{M}^{0}$ is greater than $\mathbf{M}_{c}{ }^{0}$, then $\frac{d \eta}{d U}=$ 0 at $\eta<1$. Then the flame width will not be able to increase beyond the value of $\eta$ corresponding to that at $d \eta / d U=0$. It seems that for complete combustion $M^{0}$ should be less than $\mathrm{M}^{\circ}$.

Equations [33] and [35] give the following conditions for the critical state

$$
\begin{aligned}
& \left(1-\frac{1}{2 \lambda}\right)-\frac{1}{2} \frac{U_{c}}{\sqrt{1+\lambda\left(U_{c}{ }^{2}-1\right)}} \\
& =1-\mathrm{M}_{c}{ }^{2} U_{c}\left\{1-\frac{\gamma-1}{2} \mathrm{M}_{c}{ }^{{ }^{2}}\left(U_{c}{ }^{2}-1\right)\right\}-\frac{\gamma}{\gamma-1} \\
& \left(1-\frac{1}{2 \lambda}\right) U_{c}-\frac{1}{2 \lambda} \sqrt{1+\lambda\left(U_{c}{ }^{2}-1\right)} \\
& =U_{c}-\left\{1-\frac{\gamma-1}{2} \mathrm{M}_{c}{ }^{{ }^{2}}\left(U_{c}{ }^{2}-1\right)\right\}-\frac{1}{\gamma-1}
\end{aligned}
$$

From Equations [36] and [37]

$\frac{\gamma-1}{2} M_{e} 0^{2}=$

$\frac{\lambda U_{c}+\sqrt{1+\lambda\left(U_{c}^{2}-1\right)}}{\left(\frac{\gamma+1}{\gamma-1} U_{c}^{2}-1\right)\left\{\lambda U_{c}+\sqrt{1+\lambda\left(U_{c}^{2}-1\right)}\right\}-2 \frac{\lambda-1}{\gamma-1} U_{c}}$

This equation and Equation [36] can be used to determine the relation between $M_{c}^{0}, \lambda$, and $U_{c}$ by numerical method. The Mach number $M_{c}$ corresponding to $U_{c}$ at the critical state is

$$
M_{c}{ }^{2}=\frac{M_{c}{ }^{0}{ }^{2} U_{c}{ }^{2}}{1-\frac{\gamma-1}{2} M_{c}{ }^{2}\left(U_{c}{ }^{2}-1\right)}
$$

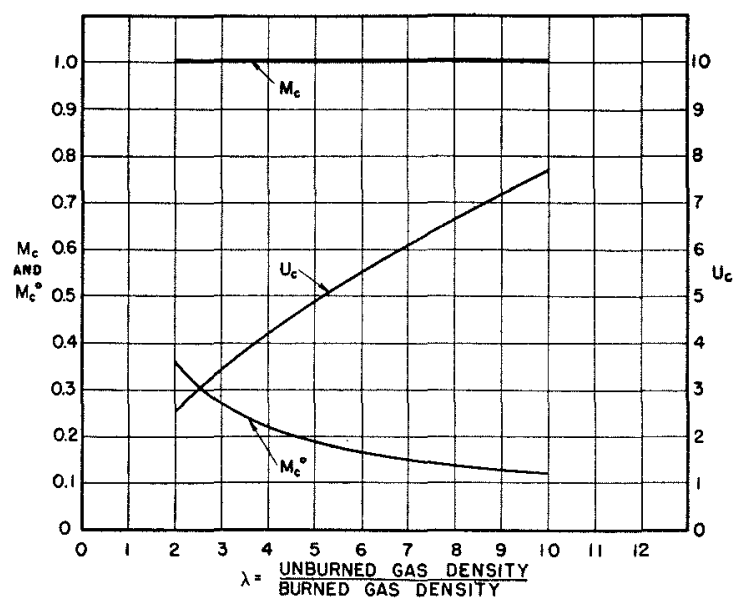

Fig. 7 Critical Condrtions for Complete Combustion at Various Density Ratios $\lambda$

The results of calculation are given in Fig. 7 where $\mathbf{M}_{c}^{0}, \mathbf{M}_{c}, U_{c}$ are plotted against $\lambda$. From the previous discussion it is seen that in order to have complete combustion, the approach Mach number $\mathbf{M}^{0}$ must be kept below the critical value $\mathbf{M}_{0}{ }^{0}$. For a heating ratio $\lambda=8, M_{c}{ }^{0}$ is only 0.15 . Then the velocity for complete combustion is below $200 \mathrm{fps}$. It is interesting to observe that the local Mach number $\mathbf{M}_{c}$, for the unburned gas at the critical state, is very close to unity but slightly supersonic.

It is unfortunate that the results of the present theoretical analysis cannot be checked with Scurlock's experiments. The few critical cases recorded by Scurlock with high inlet velocity to the combustion chamber and small flame holder are made in a combustion chamber too short to ascertain whether the flame reached the chamber wall. This is, of course, natural as Scurlock concentrated his attention on the flame-holder performance, but not in the flame-spreading and combustion efficiency. From the present investigation it would seem that the problem of flamespreading and combustion efficiency is a problem by itself, apart from the problem of flame holding, or flame initiation. In fact, one should not limit oneself only to combustion chambers of constant width, as it is evident that the way to achieve complete combustion at high approach Mach number is to use an expanding combustion chamber for reducing the flow Mach number as the combustion progresses. The present analysis is only a beginning in this problem. It serves to point out the general importance of mutual influence of flame front and the flow, and to show specifically the limitations of constant-width combustion chambers.

\section{Appendix}

Formulation of Scurlock's Problem as an Integral EquaTrON

Let the flame width increase from $\xi$ to $\xi+d \xi$ when distance downstream of the flame holder increases from $t$ to $d t$, Fig. 8 . The mass of unburned gas at $t$ is $\rho_{\mathrm{I}}(b-\xi) v$ where $v$ is the velocity of the unburned gas at $t$ (i.e., at $x=t, u_{1}=v$ ). The decrease in the mass flow of unburned gas, or the mass of gas burned $d m$ between $t$ and $t+d t$ is thus

$$
d m=-\rho_{1} d[(b-\xi) v]
$$

At section $x$, this fraction of burned mass occupies the width $d y$ with a velocity $u$ and density $\left(\rho_{1} / \lambda\right)$. From Bernoulli's equation applied between section $t$ to $x$, one has, similar to Equation [22] 


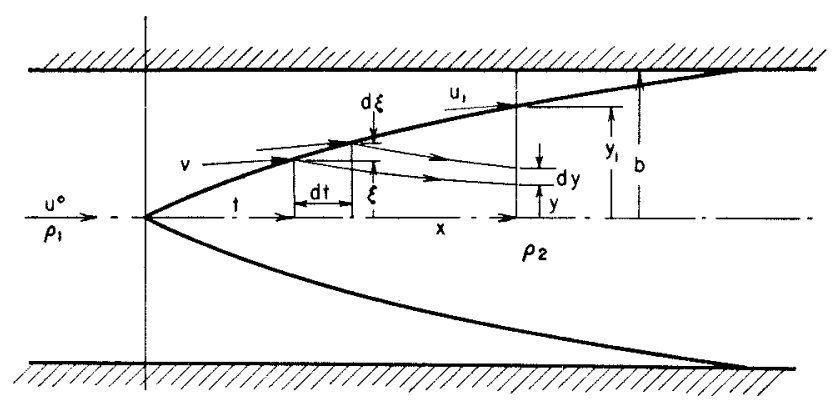

Fig. 8 Scurlock's Problem

$$
u=\sqrt{\lambda} \sqrt{u_{1}^{2}-\left(1-\frac{1}{\lambda}\right) v^{2}}
$$

By using the relation

$$
\frac{\rho_{1}}{\lambda} u d y=d m
$$

and Equation [40]

$$
d y=-\sqrt{\lambda} \frac{d[(b-\xi) v]}{\sqrt{u_{1}^{2}-\left(1-\frac{1}{\lambda}\right) v^{2}}}
$$

The streamline passing through $y=0$ corresponds to $v=u^{0}$ and the streamline passing through $y=y_{1}$ corresponds to $v=u_{1}$. Therefore, by integrating Equation [42]

$$
y_{1}=-\sqrt{\lambda} \int_{v=u^{0}}^{v=u_{1}} \frac{d[(b-\xi) v]}{\sqrt{u_{1}^{2}-\left(1-\frac{1}{\lambda}\right) v^{2}}} \ldots
$$

Result of a partial integration of Equation [43] can be written in nondimensional variables as

$$
\begin{array}{r}
\eta(U)=\frac{\lambda}{(\lambda-1)^{3 / 2}}\left\{\sin ^{-1} \sqrt{\frac{\lambda-1}{\lambda}}-\sin ^{-1} \frac{1}{U} \sqrt{\frac{\lambda-1}{\lambda}}\right\} \\
+\frac{1}{\sqrt{\lambda}} \int_{1}^{U}\left[U^{2}-\left(1-\frac{1}{\lambda}\right) V^{2}\right]^{3 / 2} \eta(V) d V \ldots \ldots
\end{array}
$$

where $V=v / u^{0}$ and $\eta(V)=\xi / b$.

Equation [44] is an integra] equation of the second kind for the unknown function $\eta(U)$. When $\lambda$ is large, a very accurate value of $\eta$ can be obtained by replacing the $\eta(V)$ in the integral of Equation [44] by the approximate value given by Equation [25]. 